\title{
Delirium in Ventilated Patients: Is ABCDEF Bundle the Solution?
}

\author{
Rajavardhan Rangappa®
}

Keywords: Bundle, Delirium care, Mechanical ventilation.

Indian Journal of Critical Care Medicine (2021): 10.5005/jp-journals-10071-23903

ICU delirium is an acute, preventable impairment in consciousness and cognition, which is characterized by disorganized thinking and fluctuation of perception over a short period. Its prevalence is as high as $50 \%$ in the ICU with a higher incidence in the critically ill ventilated patients with higher APACHE scores. ${ }^{1-3}$ Other risk factors associated with delirium include older population, cognitive impairment, terminal disease, and major surgery. ${ }^{4,5}$

Delirium can manifest as either hypoactive or hyperactive form. Hyperactive delirium manifests with restlessness, agitation, and irritability. Hypoactive delirium manifests with somnolence, lethargy, and weakness. Symptoms in both groups include altered sleep-wake cycle, inattention, impaired short-term memory, and hallucinations. Delirium is associated with increased length of stay (LOS), costs, morbidity, and mortality. ${ }^{6,7}$ It also causes emotional trauma to the patients and family. Incidence of delirium can be decreased by early identification and prevention of causative factors. Many tools are available to identify ICU delirium and one tool which has been used universally is Confusion assessment method (CAM) for ICU. CAM ICU is an effective reliable tool to assess delirium in mechanically ventilated patients. Even though the risk factors responsible for delirium are well understood and studied, there are no effective treatment options to alleviate it once established. Antipsychotics might help in decreasing the intensity of the disease. The best and only way to manage is by preventing the development of delirium by utilization of bundle concepts.

Different societies have tried to address this issue by formulating guidelines. Society of critical care medicine published clinical practice guidelines to manage pain, agitation, and delirium in the critical care unit. ${ }^{8}$ Different individuals and organizations have helped in the evolution of the delirium bundle into the current ABCDEF format. ${ }^{9-12}$

In a study published by Barnes-Daly et al., a prospective cohort quality improvement initiative, $A B C D E F$ bundle implementation in critically ill patients was associated with better survival, decreased delirium, and more coma-15. However, in this study, only $23.7 \%$ of patients received mechanical ventilation. There was a lack of studies assessing the role of the ABCDEF bundle in critically ill patients on mechanical ventilation, the subgroup with a high incidence of delirium.

Study in the current issue of IJCCM— "To determine whether implementation of a new ICU delirium prevention bundle will reduce the incidence of delirium compared to standard of care protocol in mechanically ventilated patients: randomized parallel-group trial" was a single-center prospective randomized parallel-group clinical trial conducted at the All India Institute of Medical sciences. The study included all patients aged more than 18 years and requiring mechanical ventilation for more than
Department of Critical Care Medicine, Manipal Hospital Whitefield, Bengaluru, Karnataka, India

Corresponding Author: Rajavardhan Rangappa, Department of Critical Care Medicine, Manipal Hospital Whitefield, Bengaluru, Karnataka, India, Phone: +918589969639, e-mail: rajavardhanr99@ gmail.com

How to cite this article: Rangappa R. Delirium in Ventilated Patients: Is ABCDEF Bundle the Solution? Indian J Crit Care Med 2021;25(7):743-744.

Source of support: Nil

Conflict of interest: None

24 hours and excluded patients with a history of prior neurological or psychiatric disorders, poor irreversible neurological status at the time of ICU admission, traumatic brain injury, and delirium at the time of ICU admission. It compared ICU delirium prevention bundle of care protocol and standard of care protocol.

Protocol in the intervention group included the use of earplugs and eye patch during sleeping hours, use of analgosedation, avoiding benzodiazepines, sedation using dexmedetomidine, sedation breaks, regular family contact and bonding, orienting patient with respect to time, place, and person, early mobilization, daily spontaneous awakening along with spontaneous breathing trial and early removal of catheters and lines.

The daily delirium screening was performed in the morning by an investigator using the CAM ICU tool. Fifty-seven patients were enrolled in the study, and data from 50 patients were available for analysis. The results showed a $20 \%$ reduction in the incidence of delirium in the intervention group compared to the control group (36 vs $56 \% ; p=0.156$ ) but this was statistically not significant. The 28-day mortality rate, duration of mechanical ventilation, ICU-LOS, and hospital LOS were not different between the two groups. In this study, the implementation of delirium prevention bundle led to an insignificant reduction in the incidence of newonset delirium.

This is a well-conducted study with good randomization. Independent experienced investigator who was not part of the treating team performed daily delirium screening which ruled out the possibility of inter-rater variability and the need for training. Screening of all patients by a single observer is difficult as it needs more dedication and time. It is better to train the nurses to identify delirium which is more feasible and can be done during the routine assessment. The study does not address the limitation of delirium assessment due to the language barrier with many spoken languages in India. Only the Hindi version of the CAM ICU tool is available but 
this version of the tool has not been validated. $A B C D E F$ bundle implementation is associated with multiple barriers including the need for communication between multiple disciplines.

In one of the studies, after education and training, it took 11 months for implementation of the ABCDE bundle as a standard of care. ${ }^{14}$ It may not be applicable to all the patients uniformly because of differences between patient groups, for example, neurocritical care patients, chronic alcoholics, postoperative patients, etc.

This study targeted a reduction in the incidence of delirium with multicomponent protocol by $27.1 \%$ based on one pilot study done in geriatric patients who did not require ventilatory assistance. Such a steep target may be difficult to achieve especially in the current study format with a different set of population on mechanical ventilation with longer ICU LOS. Two patients were withdrawn from the intervention group and also intended to treat analysis was not followed indicating investigator bias. This study suggests that the use of a specific bundle may improve outcomes. Further randomized multicentre trials in ventilated patients with adequate sample size including achievable targets are required to confirm the findings of this study. ${ }^{15}$

\section{OrCID}

Rajavardhan Rangappa @ https://orcid.org/0000-0001-9521-2062

\section{References}

1. Thomason JW, Shintani A, Peterson JF, Pun BT, Jackson JC, Ely EW. Intensive care unit delirium is an independent predictor of longer hospital stay: a prospective analysis of 261 non-ventilated patients. Crit Care 2005;9(4):R375-R381. DOI: 10.1186/cc3729.

2. Ely E, Inouye S, Bernard G, Gordon S, Francis J, May L, et al. Delirium in mechanically ventilated patients. JAMA 2001;286(21):2703. DOI: 10.1001/jama.286.21.2703.

3. Tilouche N, Hassen M, Ali H, Jaoued O, Gharbi R, El Atrous S. Delirium in the intensive care unit: incidence, risk factors, and impact on outcome. Indian J Crit Care Med 2018;22(3):144-149. DOI: 10.4103/ ijccm.IJCCM_244_17.

4. Inouye S. Delirium in older persons. N Engl J Med 2006;354(11):11571165. DOI: 10.1056/nejmra052321.
5. Balas M, Happ M, Yang W, Chelluri L, Richmond T. Outcomes associated with delirium in older patients in surgical ICUs. Chest 2009;135(1):18-25. DOI: 10.1378/chest.08-1456.

6. Ouimet S, Kavanagh B, Gottfried S, Skrobik Y. Incidence, risk factors and consequences of ICU delirium. Intensive Care Med 2006;33(1):6673. DOI: $10.1007 / \mathrm{s} 00134-006-0399-8$.

7. Salluh J, Wang H, Schneider E, Nagaraja N, Yenokyan G, Damluji A, et al. Outcome of delirium in critically ill patients: systematic review and meta-analysis. BMJ 2015;350:h2538-h2538. DOI: 10.1136/bmj. h2538.

8. Barr J, Kishman C, Jaeschke R. The methodological approach used to develop the 2013 pain, agitation, and delirium clinical practice guidelines for adult ICU patients. Crit Care Med 2013;41:S1-S15. DOI: 10.1097/ccm.0b013e3182a167d7.

9. Morandi A, Brummel N, Ely E. Sedation, delirium and mechanical ventilation: the 'ABCDE' approach. Curr Opin Crit Care 2011;17(1):4349. DOI: $10.1097 /$ mcc.0b013e3283427243.

10. Needham D, Korupolu R, Zanni J, Pradhan P, Colantuoni E, Palmer J, et al. Early physical medicine and rehabilitation for patients with acute respiratory failure: a quality improvement project. Arch Phys Med Rehabil 2010;91(4):536-542. DOI: 10.1016/j. apmr.2010.01.002.

11. Balas M, Vasilevskis E, Olsen K, Schmid K, Shostrom V, Cohen M, et al. Effectiveness and safety of the awakening and breathing coordination, delirium monitoring/management, and early exercise/ mobility bundle. Crit Care Med 2014;42(5):1024-1036. DOI: 10.1097/ ccm.0000000000000129.

12. Kress J, Pohlman A, O'Connor M, Hall J. Daily interruption of sedative infusions in critically ill patients undergoing mechanical ventilation. N Engl J Med 2000;342(20):1471-1477. DOI: 10.1056/ nejm200005183422002.

13. Barnes-Daly M, Phillips G, Ely E. Improving hospital survival and reducing brain dysfunction at seven california community hospitals. Crit Care Med 2017;45(2):171-178. DOI: 10.1097/ CCM.0000000000002149.

14. Balas M, Burke W, Gannon D, Cohen M, Colburn L, Bevil C, et al. Implementing the awakening and breathing coordination, delirium monitoring/management, and early exercise/mobility bundle into everyday care. Crit Care Med 2013;41:S116-S127. DOI: 10.1097/ ccm.0b013e3182a17064.

15. Malik AK, Baidya DK, Anand RK, Subramaniam R. A New ICU Delirium Prevention Bundle to Reduce the Incidence of Delirium: A Randomized Parallel Group Trial. Indian J Crit Care Med 2021;25(7):754-760. 